\title{
Magnetism in iron-nickel alloys
}

\author{
CATHERINE MCCAMMON ${ }^{1}$, QUINGGUO WEI ${ }^{2}$, STUART \\ GILDER $^{2}$
}

${ }^{1}$ Universität Bayreuth, Bayerisches Geoinstitut, Bayreuth, Germany (catherine.mccammon@uni-bayreuth.de)

${ }^{2}$ Ludwig-Maximilians-Universität München, Department für Geo- und Umweltwissenschaften, München, Germany

Iron-nickel alloys have been a subject of fascination since 1897 when Charles Edouard Guillaume discovered the near zero thermal expansion of alloys with composition around 36 mol\% nickel. These Invar ("invariable") alloys have many further anomalous properties, all linked primarily to ferromagnetism. Invar compositions may also be found in planetary cores, which may impact planetary magnetism. Despite numerous decades of research, however, many controversies regarding the properties of Invar alloys persist, including the conditions under which magnetism is lost. To address this particular controversy, we undertook a combined magnetic remanence and Mössbauer study of an Invar alloy at high pressure.

We collected Mössbauer spectra of $\mathrm{Fe}_{62} \mathrm{Ni}_{38}$ during stepwise decompression from $20 \mathrm{GPa}$ to room pressure and observed a clear loss of magnetism. We could obtain good fits to spectra with the traditional approach using a magnetic field distribution. However the mean hyperfine magnetic field decreases rapidly with increasing pressure with loss of magnetism between 10 and $15 \mathrm{GPa}$, which is inconsistent with our magnetic remanence data that show ferromagnetism to at least $16 \mathrm{GPa}$. If we instead fit Mössbauer spectra using a simple model proposed many years ago where the magnetic hyperfine field flips with a frequency comparable to the Mössbauer timescale $\left(\sim 10^{-8} \mathrm{~s}\right)$, we obtain equally good fits to the Mössbauer spectra and a hyperfine field decrease with pressure that is consistent with magnetic remanence data. More detailed comparison of Mössbauer and magnetic remanence data is underway to resolve further controversies regarding Invar alloy behaviour. Comparison of data from all methods provides insight that can be applied to planetary magnetism. 